\section{Surfaces I}

\section{Surface and Interface Crystallography}

MS12.01.01 STRUCTURE AND DISORDERING OF THE c(4X2) RECONSTRUCTION OF Ge(001) SURFACES. S. Ferrer, X. Torrelles, J.Alvarez, V. H. Etgens"; H. A. van der Vegt:** and P. Fajardo, ESRF, BP 220, 38043 Grenoble Cedex, France, *Philips Semiconductors Nijmegen, Gerstweg 2, $6534 \mathrm{AE}$ Nijmegen, The Netherlands : 4 Place Jussieu, 75005 Paris Cedex, France

The structure of the low temperature reconstruction of $\mathrm{Ge}(001)$ has been investigated by $\mathrm{X}$ ray diffraction. It consists in an array of buckled surface dimers with antiferromagnetic ordering. Upon increasing temperatures the surface disorders via a phase transition that may be described by the critical exponents of a mean field transition. The structure of the disordered phase has also been studied in detail.

MS12.01.02 MAXIMUM ENTROPY DETERMINATION OF THE ELECTRON DISTRIBUTION OF THE Si(111) 7X7 SURFACE FROM X-RAY AND ELECTRONDIFFRACTION DATA. C. A. M. Carvalho', A. Ghosh ${ }^{2}$, I. K. Robinson' ${ }^{2}$ H. Hashizume ${ }^{3}$. ${ }^{1}$ Dept. of Physics, Univ. Federal do Paranà, Brazil, 2Dept. of Phys., Univ, of Illinois, USA. ${ }^{3}$ Research Laboratory of Engineering Materials, Tokyo Institute of Technology, Japan.

The two-dimensional electron-density distribution of the Si(111) $7 \times 7$ surface has been calculated by the Maximum Entropy (ME) technique using X-ray and electron diffraction data. The maps clearly show all the 102 atoms of the Takayanagi dimeradatom-stacking fault model. The atomic coordinates defined by the density maxima are in good agreement with those obtained by a standard crystallographic leastsquares fit. It is shown that ME maps can give many more details than Fourier synthesis. A comparison between maps calculated using new $X$-ray data and maps using simulated data obtained from the DAS model shows some evidence of bonding that was absent in our previous work [C. Carvalho, $\mathrm{H}$. Hashizume, A. W. Stevenson \& I. K. Robinson: Physica B (in press)]. It is also shown that a high density level between adjacent atoms does not necessarily indicate valence charge redistribution. We discuss the reliability of the ME method and show that the calculation procedure must be continued to values of $X^{2}<1$, differently from a standard fitting process. Doubts about the validity of the method [W. Jauch \& A. Palmer: Acta Cryst. A49, 590 (1993); A50, 650 (1994)] are answered. The effect of data uncertainty in the resultant map is analysed and we propose a method to identify the features of the distributions that can be trusted. Results obtained from data sets with different numbers of reflections and data precision show that artifacts in the density distributions are caused by the data themselves and not the ME method, and that detailed information can be obtained with a relatively small number of precisely measured reflections. An example is shown where a map calculated from an $X$-ray data set with fewer reflections than an electron diffraction one in fact give better electron densities for the $\mathrm{Si}(111) 7 \times 7$ surface. The ME technique is promising to obtain detailed surface structure information.
MS12.01.03 INTERFACE STRUCTURE OF Si(111) $\sqrt{3} \times \sqrt{3}$ Ag STUDIED BY GRAZING INCIDENCE X-RAY DIFFRACTION. Koichi Akimoto, Melania Lijadi, Takayuki Susami, and Ayahiko Ichimiya, Department of Quantum Engineering, Nagoya University, Furo-cho, Chikusa-ku, Nagoya 464-01, Japan

Buried interface structures of $\mathrm{Ag} / \mathrm{Si}(111) \sqrt{3} \times \sqrt{3}-\mathrm{Ag}$ and $\mathrm{Ge} /$ $\operatorname{Si}(111) \sqrt{3} x \sqrt{3}-\mathrm{Ag}$ were studied by grazing incidence $X$-ray diffraction using synchrotron radiation. A Si(111) $\sqrt{3} x \sqrt{3}-\mathrm{Ag}$ surface was obtained by depositing $1 \mathrm{ML}\left(7.8 \times 10^{\left.14 / \mathrm{cm}^{2}\right)}\right.$ of Ag on a $\mathrm{Si}(111) 7 \times 7$ reconstructed surface heated up to $400-500^{\circ} \mathrm{C}$ in an UHV chamber operating in the 10-10 Torr range. On that surface, $\mathrm{Ag}$ $(8 \mathrm{~nm})$ and $\mathrm{Ge}(6 \mathrm{~nm})$ were deposited at room temperature. The Xray diffraction measurements using synchrotron radiation were carried out at the BL-9C of the Photon Factory, Japan. During measurements, we covered the sample with an evacuated dome in the $10^{-2}-10^{-3}$ Torr range.

For the sample of $\mathrm{Ag} / \mathrm{Si}(111) \sqrt{3} \mathrm{x} \sqrt{3}-\mathrm{Ag}$, a $(4 / 3,1 / 3) \mathrm{X}$-ray diffraction peak was observed, while the $(4 / 3,1 / 3)$ peak was not observed for the sample of $\mathrm{Si}(111) \sqrt{3} \mathrm{x} \sqrt{3}-\mathrm{Ag}$ with native oxide layers. Therefore, we conclude the $\sqrt{3}$ structure remained at the $\mathrm{Ag} / \mathrm{Si}(111)$ interface. The surface $\mathrm{Si}(111) \sqrt{3} 3 \mathrm{~V} 3 \mathrm{3}-\mathrm{Ag}$ structure was normally explained by the honeycomb-chained-trimer (HCT) model. The HCT model should give rise to the comparably intense $(2 / 3,2 / 3)$ peak. However, we did not observe the $(2 / 3,2 / 3)$ peak for the sample of $\mathrm{Ag} / \mathrm{Si}(111) \sqrt{3} \times \sqrt{3}-\mathrm{Ag}$. This fact suggest that the $\sqrt{3}$ structure at the $\mathrm{Ag} / \mathrm{Si}(111)$ interface is a different type from the surface $\sqrt{3}$ structure.

For most samples of $\mathrm{Ge} / \mathrm{Si}(111) \sqrt{3} \mathrm{x} \sqrt{3}-\mathrm{Ag}$, X-ray diffraction peak from the $\sqrt{3}$ structure was not observed. However, the in-plane 220 diffraction peak of the Ag(111) film was found. The correlation length derived from a peak width was about $3 \mathrm{~nm}$. The $\mathrm{Ag}(111)$ film is thought to exist under the Ge islands.

MS12.01.04 DEFECTS IN THE RECONSTRUCTED (111) AND (-1 -1 -1) SURFACES OF InSb. H. Schulz, H. Vogler, H.L. Meyerheim, W. Moritz Institut für Kristallographie und Mineralogie, Universität München. Theresienstr. 41, 80333 München, Germany

The 3D-structures of the (111) and $(\overline{111})$ surfaces of InSb have been determined by grazing incidence $x$-ray diffraction using superstructure reflections up to high exit angles. Both surfaces exhibit a reconstruction. The In-terminated (111) surface shows a $(2 \times 2)$ reconstruction and the Sb-terminated (111) surface has a (3x3) structure,

The (2x2) reconstruction is characterised by a In-vacancy and at rearrangement of the bonds of the surface atoms which can be explained, by rehybridisation and depolarisation [1]. Similar structures have been found on other III/V semiconductor surfaces. We present here a new 3-D x-ray structure analysis using 29 symmetrically independent in-plane reflections and 244 out-of-plane reflections from superstructure rods and truncation rods. The results clearly favour the model with statistically distributed Sb vacancies in addition to the In vacancies [2]. The density of the Sb vacancies is about 0.07 monolayers, i.e. in one of three or four $(2 \times 2)$ unit cells the In vacancy is occupied and an $\mathrm{Sb}$ is missing. The existence of Sbvacancies is not expected on this surface from total energy calculations and also from the observation that by the defects the electron counting rule is violated. The existence of defects is discussed and compared to the $(3 \times 3)$ reconstruction of the $(\overline{111})$ surface. In this surface 6 of the 9 dangling bonds in the $(3 \times 3)$ unit cell are saturated by rings of 6 atoms. The rings are statistically distributed and oriented. The results of the X-ray structure analysis agree well with STM images of the same surface [3].

[1] J. Bohr, R. Feidenhans'l M. Nielsen, M. Toney, R.L. Johnson and I.K. Robinson, Phys. Rev. Letters 54 (1985) 1275.

[2] A. Belzner, E. Ritter, and H. Schulz, Surf. Sci. 209, (1989) 379.

[3] J. Wever, H.L. Meyerheim, W. Moritz, V. Jahns,, D. Wolf, H. Schulz, L. Seehofer and R.L. Johnson, Surf. Sci Lett. 321 (1994) L225. 